\title{
Pensamiento complejo y creatividad
}

Fecha de recepción: 9 de septiembre de 2016

Fecha de aprobación: 9 de diciembre de 2016 Pp. 7-26

\author{
Beatriz Cecilia Ruiz Lara ${ }^{1}$ \\ Universidad EAN \\ becelara@hotmail.com \\ Luis Carlos Torres Soler ${ }^{2}$ \\ Universidad Cooperativa de Colombia \\ lctorress@gmail.com
}

DOI: https://doi.org/10.21158/23823399.v4.n2.2016.1625

Cómo citar este artículo/ To reference this article/Comment citer cet article/ Para citar este artigo: Ruíz, B. C. y Torres, L. C. (2016). Pensamiento complejo y creatividad. Revista Ontare, 4(2), 7-26. DOI: https://doi.org/10.21158/23823399.v4.n2.2016.1625.

\section{Resumen}

Este artículo es una reflexión sobre elementos teóricos del conocimiento que se generan desde la complejidad, el pensamiento complejo y los retos de la educación superior en una sociedad que cada día demanda nuevas estrategias y el desarrollo de un pensamiento que permita comprender y actuar sobre una parte, sin perder las implicaciones que se generan con el contexto y superar las barreras que nuestra percepción limitada nos impone, teniendo como herramienta la creatividad para abordar una misma realidad desde diferentes posiciones y aplicando estrategias que permiten potenciarla. Para la comprensión del proceso creativo se requieren miradas en distintas dimensiones, interrelacionar diversos aspectos, determinar cómo los procesos educativos transdisciplinares tejen los conocimientos y pueden desarrollar la creatividad latente en cada persona.

\section{Palabras clave}

Creatividad, educación, pensamiento complejo, transdisciplinariedad.

\footnotetext{
${ }^{1}$ Docente Investigadora Universidad EAN y Escuela de Posgrados Fuerza Aérea Colombiana EPFAC. Doctorante Pensamiento Complejo Multiversidad Mundo Real Edgar Morin. ORCID: http://orcid. org/0000-0003-0266-2473

2 Matemático, MSc. Ingeniería de Sistemas, MA. Ciencias de la Educación. Doctorante en Pensamiento Complejo, Multiversidad Mundo Real Edgar Morin. ORCID: http://orcid.org/0000-0001-6756-4984
} 


\title{
Complex thinking and creativity
}

\begin{abstract}
This article is a reflection on the theoretical elements of knowledge that are generated from the complexity, the complex thinking and the challenges of the higher education in a society that day by day is demanding for new strategies and the development of a thought that allows to understand and to act on one part, without losing the implications that are generated with the context, and overcome the barriers that our limited perception imposes on us, having the creativity as a tool to approach the same reality from different positions and applying strategies that allow it to be strengthened. In order to understand the creative process, it is necessary to look at different dimensions, to interrelate different aspects, to determine how the transdisciplinary educational processes weave knowledge, and to develop latent creativity in each person.
\end{abstract}

\section{Keywords}

Creativity, education, complex thinking, transdisciplinarity.

\section{Pensée complexe et créativité}

\section{Résumé}

Cet article offre une réflexion sur les éléments théoriques de la connaissance générés par la complexité, la pensée complexe et les défis de l'éducation supérieure face à une société exigeant constamment de nouvelles stratégies pour permettre de développer une pensée offrant compréhension et action mais conservant simultanément les implications générées par le contexte sans outrepasser les limites imposées par notre perception. La créativité se trouve être l'outil pour aborder cette réalité sous différents points de vue en utilisant les stratégies qui permettent son renforcement. Pour bien comprendre le processus de création, différents angles d'analyse sont requis pour mettre en relation les divers aspects du processus et ainsi déterminer de quelles manières les processus éducatifs transdisciplinares tissent et mettent en relation les connaissances développant la créativité latente de chaque individu.

\section{Mots clefs}

Créativité, éducation, pensée complexe, transdisciplinarité. 


\section{Pensamento complexo e criatividade}

\section{Resumo}

Este artigo é uma reflexão sobre os elementos teóricos do conhecimento que se geram a partir da complexidade, do pensamento complexo e dos desafios da educação superior em uma sociedade que a cada dia exige novas estratégias e o desenvolvimento de um pensamento que permita compreender e agir sem perder as implicações que se estabelecem com o contexto, superando as barreiras que nossa percepção limitada nos impõe, tendo como ferramenta a criatividade para abordar uma mesma realidade a partir de diferentes posições e aplicando estratégias que permitem torná-la sempre mais potente. Para o entendimento do processo criativo requerem-se olhares em diferentes dimensões, inter-relacionar diversos aspectos, determinar como os processos educativos transdisciplinares tecem os conhecimentos e podem desenvolver a criatividade latente em cada pessoa.

\section{Palavras-chave}

Criatividade, educação, pensamento complexo, transdisciplinaridade. 


\section{Introducción}

a educación universitaria enfrenta, en la era de la información, nuevos Uretos impuestos por la globalización de las economías, el acelerado desarrollo de las tecnologías y las constantes transformaciones sociales que le exigen adaptar y adaptarse a los diversos procesos de aprehensión de conocimientos, que sumen a la formación de seres que con sabiduría logren hacer de sus contextos mejores espacios para que la vida florezca.

Esto obliga a que los profesionales posean competencias blandas: sean empáticos, comunicadores asertivos, constructores aún con las diferencias, cooperativos, comprometidos con sus equipos, deben ostentar conocimientos técnicos en sus áreas de desempeño, pero con capacidades interdisciplinarias dirigidas hacia la transdisciplinariedad, innovadores y creativos, de ahí que los procesos formativos deben orientarse hacia aprendizajes multidimensionales que los conduzca a resolver problemas con una visión total y parcial al mismo tiempo, actuar en lo local mirando lo global, con pensamientos abiertos y flexibles, apoyados en el uso de tecnologías de la información y las comunicaciones (TIC), de tal forma que los estudiantes aprendan, critiquen y gestionen conocimiento por medio de estas.

Es decir, en todos los ámbitos educativos, en especial en la universidad, se deben realizar cambios con nuevas propuestas, tanto en el currículo como en la metodología. Por tanto, la educación hoy día, en esta era de la información, en la que las TIC facilitan que los estudiantes adquieran toda la información que requieren de manera oportuna, ya no debe poseer rígidos sistemas curriculares, mucho menos fragmentar y condicionar el contenido que pueden aprender y, en algunos casos, no permiten encontrar respuestas diferentes de las tradicionales cuando se aborda un problema. Hay que dejar a un lado la transmisión de un conocimiento disciplinar, inacabado, por uno transdisciplinar, en el que los estudiantes trabajen en 
equipos interdisciplinarios. Por tanto, el pensamiento debe proyectarse a otras dimensiones, generar un pensamiento complejo.

De este modo, el proceso creativo requiere una mirada amplia en cada una de las situaciones que se abordan, en particular con diferentes enfoques, realizando analogías, hallando detalles y resultados nuevos, ojalá innovadores, pues las demandas de la nueva sociedad son cada vez más complejas.

El escrito toma en contexto el pensamiento complejo y la creatividad en un todo, pues se considera que las interacciones posibles entre estos son fuertes, continuas y dinámicas, generando un bucle de retroalimentación positiva entre el pensamiento complejo y la potenciación de la creatividad que se concretan en un sujeto creativo.

\section{Pensamiento complejo}

T a creatividad, la innovación y la invención son productos de unsadores complejos como Leonardo da Vinci, quien abordó transdisciplinarmente el conocimiento, superando las barreras disciplinares de los saberes, lo que condujo a grandes inventos con magníficos resultados. Muchas circunstancias debieron favorecer sus logros, pero quizá su proceso creativo tuvo gran relevancia; ¿qué lo hacía tan especial?: observaba su entorno, la naturaleza, desde diferentes perspectivas, iba más allá de lo evidente en la búsqueda de solucionar problemas, creando o mejorando situaciones reales o imaginarias.

Examinaba con prismas diversos para ampliar los escenarios de posibilidades, sus conocimientos, permitiéndole reflexionar simultáneamente sobre el todo y las partes, promoviendo los retos, la perseverancia, advertía las relaciones entre los elementos y sus sistemas, provocaba ideas divergentes: transformaba, inventaba. 
De igual forma Edgar Morin, filósofo francés considerado el pensador del siglo XXI, y padre del pensamiento complejo, propone la transformación de la educación para que desarrolle un pensamiento capaz de interconectar distintas dimensiones, mediante una visión global, sin descuidar lo particular; que integre, no que separe el objeto de estudio [Morin, 1999].

La fragmentación de los saberes y el aislamiento entre las disciplinas se palpan en el contexto educativo, desde los procesos en el aula hasta la vida misma. Los currículos, la evaluación, los procesos de enseñanza y aprendizaje siempre permanecen con una visión disciplinaria (Morin, 1999a). La realidad educativa muestra la fragmentación y la disyunción entre procesos de la enseñanza, el aprendizaje y la evaluación; esto produce problemas teóricos y prácticos, visibles en su contexto por la medición, según un enfoque cuantitativo. Esta fragmentación y esta realidad educativa involucran pensamiento.

Al estudiar cómo comprender el conocimiento, que genera un proceso cognitivo, se advierte que es producido, desplegado, comunicado, transformado y aplicado en el pensamiento (Morin, 2001b). El pensamiento, considerado en su movimiento de llegar a ser (y no simplemente en su contenido de imágenes e ideas relativamente bien definidas), es, ciertamente, el proceso en el cual tiene su real y concreta existencia el conocimiento.

El pensamiento es, en esencia, la respuesta activa de la mente en cada fase de la vida. Se incluye en el pensamiento respuestas intelectuales, emocionales, sensitivas, musculares y físicas. Estos son aspectos de un solo proceso indisoluble. Tratarlos separadamente produce fragmentación y confusión. Todos constituyen un proceso de respuesta de la mente a cada situación real, y tal respuesta, a su vez, produce una nueva contribución. 
El pensamiento, considerado como la respuesta de la mente, es básicamente mecánico en su modo de operar, es la repetición de alguna estructura previamente existente, traída por la mente, o es cierta combinación, adaptación y organización de lo guardado en subsiguientes estructuras de ideas y conceptos, categorías, etcétera. Se forman combinaciones que pueden poseer cierta especie de novedad, resultado de la interacción fortuita de elementos de la mente, pero tal novedad sigue siendo esencialmente mecánica. En ese proceso mecánico no hay razón intrínseca para que los pensamientos que surjan sean relevantes o adecuados a la situación real que evoca; esencialmente, una situación es un acto de percepción, y luego se expresa en pensamiento.

El pensamiento que puede ser una alternativa es el complejo, pues proyecta que se desarrolle una estrategia reflexiva y no reductiva, incorpora multiplicidad de elementos y relaciones para el estudio de las diversas situaciones, incluyendo al sujeto observador como una parte de la misma situación. Es decir, es incluyente, valora la diversidad y distingue la individualidad (Morin, 1999a).

El pensamiento complejo es producto de un proceso sistémico que no contempla la causalidad lineal, sino una causa que produce un efecto, y el efecto actúa sobre la causa: un bucle retroactivo, que conduce a una autoorganización. Pero las dinámicas que se forman en ese bucle están totalmente reguladas. Morin considera, en los fenómenos, lo antagónico como complementario y estima que la incertidumbre siempre está presente (citado en Soto, 1999); por tanto, debe abordarse para enfrentar el azar, lo que conduce a que surja la creatividad, la imaginación, la gestión del conocimiento.

Sus características apuntan a que el estudiante perciba una totalidad, que su aprendizaje se produzca por factores del entorno conjugados con sus capacidades cognitivas que, por naturaleza son transversales, proveen autoorganización y adaptación para enfrentar el caos y la incertidumbre. No obstante, variadas características de todo individuo se presentan en 
el proceso creativo que, muchas veces, se realiza de modo inconsciente, pero que de manera intuitiva no deja de persistir en las metas planeadas.

El docente aclara en su clase, entre otras cosas, puntos claves de una temática; sin embargo, no existe apertura de espacios para una experiencia formativa y abierta, que implique varias posibilidades, que responda a preguntas o cuestionamientos vitales, que sugiera alternativas de solución que el estudiante piensa y evalúa. Y la evaluación tiene un enfoque instrumental, riñendo con procesos que se encaminan a fortalecer la formación integral, a potenciar la creatividad y al desarrollo del pensamiento complejo.

La dificultad del pensamiento complejo es que debe afrontar lo entramado (el juego infinito de interretroacciones), la solidaridad de los fenómenos entre sí, la bruma, la incertidumbre, la contradicción. No obstante, se pueden elaborar elementos conceptuales, principios, para una aventura que lleve a una transformación de los procesos educativos, en particular involucrando el paradigma de complejidad y desarrollando estrategias para potenciar la creatividad empleando un pensamiento complejo.

\section{El sujeto creativo}

7 studiar el proceso creativo no es sencillo. Existen múltiples factores: Cinterrelaciones y elementos. El principal es el entorno, el que inhibe o impulsa; el que motiva o desmotiva, el que separa o integra; el que promueve que el conocer sea un proceso sistémico, el que proyecta la observación de los detalles en las distintas situaciones, el que conduce a que se acoja el individuo como parte de una acción investigadora. 
El entorno, más precisamente el proceso educativo, conduce a que el sujeto se cuestione libre y constantemente, repensando, reflexionando, criticando el saber que recibe, que lo introduce sin temor a que cometa errores, a hallar contradicciones o incertidumbres con el fin de abordarlas de la mejor manera. Es decir, como lo indica Morin (1999a), los procesos educativos, en particular los docentes, deben sentir la necesidad de entregarles a los estudiantes más incertidumbres y menos certezas, guiarlos para que hallen problemas y no se les entreguen ejercicios que, particularmente, solo tienen una respuesta. Todo esto con el fin de estimular la curiosidad, la imaginación, la observación, la búsqueda incesante de conocimiento, que se motive a la experimentación, a descubrir lo que está oculto; esto es una especie de energía que impulsa al estudiante a que sienta deseos por investigar y gestionar conocimiento.

En algunos momentos, el estudiante está interesado en áreas diferentes a las de su formación, lo cual permite entrar en ámbitos distintos en los que podría crear ingeniosos productos o mejorar unos ya existentes (De la Torre, 2008). En general, ser creativo tiene multiplicidad de intereses, pero debe rescatarse que se realizan transformaciones a esos mismos intereses, pues de alguna forma se mejora la motivación para dejarse fascinar y absorber por distintos aspectos en la realidad, transfiriendo luego una comprensión enriquecida de lo percibido a objetos concretos. Por tanto, una parte de la pedagogía, para quienes se dedican a la docencia, es incitar a los estudiantes a ser creativos.

Da Vinci dijo: «Todo nuestro conocimiento tiene su origen en los sentidos» (Gardner, 2002). Esto lleva a pensar que la percepción tiene gran importancia en el proceso de aprendizaje; en otras palabras, los sentidos desempeñan un papel importante para comprender qué sucede en el entorno, y la educación debería reflexionar en cómo potenciarlos en lugar de emplear al máximo la memoria. La vista debe ser aguda, para que una buena observación determine detalles, amplíe el proceso de conocer, pues no se puede llegar a comprender un objeto si solamente se mira en una sola dimensión, desde un solo ángulo; debe analizarse desde 
todos los ángulos posibles y con diferentes enfoques. El oído debe ser agudo para escuchar los murmullos que se suscitan en el entorno, que pueden dar buen conocimiento por la energía que los genera.

Las ideas, los procesos o productos que generan los sentidos de una persona creativa, en general, no están guiados por teorías preestablecidas ni métodos que se conozcan; son producto de la observación y la reflexión; surgen con una visión integradora de diferentes áreas. Es un producto transdisciplinar.

Entonces, para ser creativo, el conocimiento es fundamental. Este permite comprender el orden/desorden del mundo, el mundoy las diferentes situaciones, las leyes que podrían determinar un comportamiento. Un aspecto fundamental para la interpretación de la naturaleza, del lugar de la humanidad en la globalidad, del sujeto en el orden/desorden que existe, es reflexionar y vincular distintas áreas con el fin de que el enfoque sea amplio y la visión sea desde diferentes ángulos.

En el aula, el análisis pedagógico de la creatividad incluye, necesariamente, la motivación que el docente le provee al estudiante; es decir, esta se halla en directa relación con los intereses del individuo. La motivación que pueda producir el entorno social, llamada motivación extrínseca, abre la mente para impulsar la generación de diferentes ideas que vienen a la mente. El entorno social lleva a todo individuo a diseñar una gran variedad de objetos, sistemas y elementos que podrían ser estratégicos para afrontar distintas situaciones, para solucionar problemas, o ser novedosos.

Sin embargo, también es muy importante la motivación intrínseca: esa preocupación que tiene el ser por resolver algunas deficiencias en la sociedad, y que de alguna manera serían fundamentales. Así, el individuo se interesará por la creación de diversos mecanismos. Para lograr que todo sea posible, utilizará diversos tipos de energía a fin de que la mezcla 
lo lleve a distintos estadios en que trabaja, descansa, reflexiona y sueña, y así construir cosas originales (Csikszentmihalyi, 1998).

Como toda persona tiene sueños, estos deben trasladarse a lo real; primero en papel, luego, si es posible, diseñar lo soñado. Entonces, diseñar varios modelos que podrían estar fuera del concepto que establece el mismo conocimiento puede ser rasgo de creatividad (Torres, 2012); es decir, puede generar algo que aún no se conoce; no importa que pueda rechazarse por la comunidad.

Además, muchas veces se desconocen avances tecnológicos que podrían, en algún momento, favorecer el pensar para proveer ideas creativas $^{3}$, pues tras una idea puede surgir otra y otra, y, por tanto, llegar a generarse productos o procesos innovadores que ayuden a estar a la vanguardia de la competitividad ${ }^{4}$. Los avances tecnológicos son precisamente las herramientas computacionales que es posible adquirir a cada instante, utilizar de forma adecuada y ayudar al desarrollo de distintas habilidades y capacidades que podrían estar inhibidas por algún motivo.

Por ejemplo, desde mediados del siglo XX, varios investigadores plantearon la idea de construir una máquina que pudiera emular los procesos cognitivos que realiza el ser humano; en pocas palabras, que

\footnotetext{
La creatividad es la capacidad que tiene el ser humano para comprender la realidad oculta y generar y expresar nuevas ideas, que adquieren gran valor y significado (Letelier, 2001). Las ideas creativas son la verdadera fuente de poder en cualquier organización moderna para estar en la competitividad del mercado (Fourez, 2005). Las ideas creativas son el ADN de aquello que podría ser muy valioso (Maslow, 1990).

4 Existen diferentes conceptos sobre innovación, mas hoy día ante la globalización de las economías, en las empresas la innovación resulta imprescindible para la competitividad; esta puede verse en productos, servicios o métodos (procesos). Innovación, en sí es producir algo totalmente nuevo (López, 1999). Sin embargo, algo totalmente nuevo podría ser aquello que resulta de hacerle modificaciones a algo ya existente, de hacer servir algo para tareas que no fueron concebidas.
} 
la máquina tuviese inteligencia (artificial) para que razonara y tomara decisiones autónomamente. Algo se ha logrado. Por lo menos, el computador es más veloz que el cerebro, mucho más veloz; pero no tiene capacidad de pensar, razonar o reflexionar sobre algo. Solamente procesa datos y, como resultado, produce gran cantidad de datos según los parámetros estipulados (programa); sin embargo, la creatividad del ser humano provee ideas para que esas máquinas sean realidad, para que sea posible diseñar programas que muestren las capacidades cognitivas del ser humano, es decir, capacidad para realizar síntesis de documentos, diseñar mecanismos, realizar pinturas y partituras de melodías (Veraldi, 1998); y en esa búsqueda persistente de algo se mejora la motivación y, seguro de esta forma, el cerebro genera ideas originales con gran flexibilidad y fluidez, propiedades que deben estimularse para ser considerado creativo (López, 2008).

En el proceso creativo, las características observables que pueden potenciarse en el aula son: apertura, curiosidad, fluidez, flexibilidad, observación, sensibilidad para hallar y definir los problemas, sus causas y efectos; habilidades mentales para considerar los riesgos que suceden, pero, a la vez, habilidad para aceptar retos, cualquiera sea su contexto. Esto induce a reflexionar en torno a que la formación en el aula debe transformarse para dejar un poco la función memorística que tiene el individuo según la concepción del pensamiento reduccionista y lineal, y dirigirse a potenciar esas características necesarias para que surjan procesos creativos (Torres, 2011). 


\section{La complejidad}

Coy, a cada momento, se puede adquirir información desde los _ más recónditos lugares, de los más variados temas en un tiempo oportuno, pero ¿qué hace esa información en la mente? ¿Se gestiona conocimiento? ¿Se establecen relaciones para aclarar aspectos de un fenómeno o problema? No se sabe. Es muy difícil precisar una respuesta adecuada por la complejidad de la sociedad, del cerebro, del conocimiento; además, aunque se distinguen características del proceso creativo, para cuyo análisis, debido a su complejidad, deben incluirse muchos factores: elementos, relaciones, propiedades.

El método científico, además de reinar por varios siglos, siempre llevó a determinar que para conocer sobre un sistema se requería dividirlo en sus partes, analizar cada una por separado y luego relacionar el conocimiento alcanzado para comprender de forma total el sistema. Sin embargo, la observación del ser humano le hizo reflexionar y pensar que existen sistemas que poseen propiedades que no están en ninguna de las partes; que esas propiedades surgen por la misma interacción entre algunas de ellas; es decir, el sistema puede tener propiedades que no están en ninguna parte (Torres, 2007). A partir de esto, surge la teoría de sistemas generales o TGS, aplicada a sistemas de índole biológico, ecosistemas y de la vida, pero sigue su avance hasta establecer los principios del pensamiento sistémico 5 . Uno de ellos es que la suma de las partes es menor que el todo. Es decir, el pensamiento sistémico determina

El pensamiento sistémico se aplica esencialmente en sistemas de índole social, es decir, en fenómenos que suceden en una organización, cuyo comportamiento depende del accionar diferente de las distintas personas en ella, del contexto en que se halla, del tipo de producto o servicio que presta, del mercado a que ha tenido capacidad de acceso y de otros factores internos y externos que no son a simple vista posible detectarlos, mucho menos con un solo enfoque o visión, porque hay complejidad. 
que el sistema (todo) debe estudiarse en su conjunto y no por partes; además, en su propio entorno. Puede que en algunos casos sea necesario estudiar la parte para comprender las interacciones que existen, cómo es ella y qué propiedades posee, entre otras cosas.

El pensamiento sistémico no es una metodología, quizá tampoco una disciplina. Es un enfoque que permite analizar sistemas de cierto tipo en los que el método científico, basado en la separación de las partes, no es adecuado para aplicarse, puesto que es necesario considerar el entorno y al mismo observador que busca comprenderlo. Su enfoque sugiere qué es importante del sistema, qué interacciones existen entre las partes, las propiedades emergentes que surgen a partir de esas interacciones e indica qué interesa, ya que el todo es más que la suma de las partes.

Distintos enfoques surgen, respecto a la complejidad y a la creatividad, que en algunos contextos se llaman paradigmas, pero no son más que modelos conceptuales que tienen el propósito de permitir entender qué es, para qué sirve, dónde se halla, cómo estudiarse. Los paradigmas surgen en procura de mejorar o transformar teorías que podrían ser válidas en un contexto muy limitado.

La complejidad del proceso creativo no puede apreciarse, pues en él existen variedad de interacciones, datos, experiencias, conocimiento y reflexiones, entre otros elementos, los cuales no deben separarse. Son un todo complejo. Deben mirarse en distintas dimensiones, con variados enfoques y una visión globalizadora.

En los distintos campos del saber resulta estrictamente necesario vincular la observación al entorno con la visión imaginaria, sin límites ni restricciones, considerando todos los ángulos posibles, para conectarlos con las imágenes mentales, por analogías, similitudes con semejanzas o contradicciones. 
Entonces, ¿qué es la complejidad? A primera vista, la complejidad es un tejido (complexus: lo que está tejido en conjunto) de constituyentes heterogéneos inseparablemente asociados (Torres, 2007): presenta la paradoja de lo uno y lo múltiple. Con mayor atención, la complejidad es el tejido de eventos, acciones, interacciones, retroacciones, determinaciones, azares, que constituyen nuestro mundo fenoménico (Morin, 2002). La complejidad se presenta con los rasgos inquietantes de lo enredado, de lo inextricable, del desorden, de la ambigüedad, de la incertidumbre. De allí la necesidad, para el conocimiento, de poner orden en los fenómenos, rechazando el desorden, descartando lo incierto; en otras palabras, selecciona los elementos de orden y de certidumbre, quita la ambigüedad, aclara, distingue, jerarquiza. Pero tales operaciones, necesarias para la inteligibilidad, corren el riesgo de producir ceguera e ilusiones, de eliminar caracteres de lo complejo; por ejemplo, donde el orden y el desorden conviven para establecer la organización adecuada.

Pero la complejidad vuelve a las ciencias por la misma vía que se fue (Morin, 2000). El desarrollo de la física, que se ocupó de revelar el orden impecable del mundo, su determinismo absoluto y perfecto, su obediencia a una ley única y su constitución de una materia simple primigenia (el átomo), se ha abierto finalmente a la complejidad de lo real (Torres, 2007). Se ha descubierto en el universo físico un principio hemorrágico de degradación y de desorden (segundo principio de la termodinámica); luego, en el supuesto de la simplicidad física y lógica, se descubre la extrema complejidad microfísica; la partícula no es un ladrillo primario, sino una frontera de la complejidad tal vez inconcebible; el cosmos no es una máquina perfecta, sino un proceso en vías de desintegración y de organización.

También se evidencia que la vida no es una sustancia, sino un fenómeno de autoeco-organización extraordinariamente complejo que produce la autonomía. Es decir, se evidencia que los fenómenos antroposociales no podrían obedecer a principios de inteligibilidad menos complejos que aquellos requeridos para los fenómenos naturales. Se debe 
afrontar la complejidad antroposocial en vez de disolverla u ocultarla, abordarla en su contexto, porque en cada contexto existen diferentes elementos, interacciones, retroacciones.

El propósito de las ciencias sociales es contextualizar posturas idealistas o materialistas, con distintas propuestas para su quehacer, con ciertos criterios de validez del conocimiento. Al respecto se visibiliza una separación e incomunicación de las ciencias y las disciplinas, para comprender, significar e interpretar un fenómeno educativo, presentándose un distanciamiento entre el objeto por conocer y el sujeto cognoscente, lo que suscita problemas de orden cognitivo, ético y moral, y emerge tensiones desde lo humanista y lo científico que generan un cruce de racionalidades en el contexto educativo y logran que este sea más complejo y se produzcan relaciones de poder de los docentes y los estudiantes, represiones, castigo y, desde luego, temor y miedo en la forma de abordar la búsqueda de una respuesta a los problemas que se presentan.

Los principios de la complejidad inducen a constantes cambios. Por ello, el ser creativo se considera muchas veces disperso, porque realiza, quizá de forma inconsciente, un bucle retroactivo (Morin, 2001), donde la percepción y el análisis de un problema no siguen un proceso lineal, sino que sucede un ir y venir, con periodos de descanso y reflexión; sin eliminar elementos aparentemente ajenos al problema, sino haciéndolos parte del análisis $\mathrm{y}$, desde luego, parte del todo. Así, todo proceso se enriquece por nuevas percepciones, lo que lleva a la generación de ideas originales que, de alguna forma, son novedosas e influyen en el entorno para que este mismo impulse a que se hagan realidad (Torres, 2011). 


\section{Conclusiones}

T1 proceso creativo es un fenómeno complejo, con elementos en Cinteracción continua, que induce a la comprensión integral de un tema, a identificar los procesos mentales que intervienen en las concepciones, y a establecer estrategias que permitan al estudiante un aprendizaje significativo.

Un resultado podría considerarse inconcluso, disperso, ambiguo, pero, como sucede dentro del bucle recursivo, se siguen generando múltiples ideas, que se evalúan y mejoran para terminar el camino. Es decir, debe comprenderse la complejidad de los fenómenos que se analizan, utilizar la observación y el entorno de manera sistémica, y no seguir un proceso lineal.

El proceso creativo tiene muchos componentes que pueden potenciarse en el aula. La curiosidad y la motivación requieren actividades que sean significativas para los estudiantes y de espacios que faciliten la reflexión, la autoevaluación, el aprendizaje a través de los sentidos y la imaginación.

El ambiente del aula para la creatividad debe ser receptivo a las nuevas ideas, no rechazar ninguna por más loca que parezca. Esto ayuda a los estudiantes a desarrollar la autoestima y la automotivación, la apertura mental y la visión globalizadora, realizar conexiones insospechadas y fuera de lo normal, construir analogías poco usuales que globalmente muestren aspectos de la creatividad.

El conocimiento así considerado permite reconocer los fenómenos multidimensionales y transdisciplinarios. El ambiente en el aula debe ser tal que, en vez de aislar y fragmentar, respete la diversidad e integre lo único que existe; además, que fomente la autonomía. 
Los cambios en los procesos educativos, en particular en la universidad, deben orientarse hacia una reforma del pensamiento, al desarrollo del pensamiento complejo, que les permita a los futuros profesionales actuar creativamente en sus labores. Podría ser siguiendo lo propuesto por Morin (2001a), una reforma educativa que se soporte en la teoría de la complejidad, que se encargue de hacer crítica a la fragmentación y disyunción del conocimiento; además, que se construyan espacios para un aprendizaje significativo, para potenciar la creatividad, las competencias blandas y otras capacidades que posee la persona. 


\section{Referencias}

Csikszentmihalyi, M. (1998). Aprender a fluir. Barcelona, España: Kairos.

De La Torre, S. (2008). Creatividad aplicada: recursos para una formación creativa. Madrid, España: Escuela Española.

De La Torre, S. (1982). Educar en creatividad. Madrid, España: Narcea.

Fourez G. (2005). La construcción del conocimiento científico. 3a ed., Madrid, España: Narcea.

Gardner, H. (2002). Mentes creativas: una anatomía de la creatividad. Barcelona, España: Paidós Ibérica.

Gardner, H. (2011). Las cinco mentes del futuro. Barcelona, España: Paidós Ibérica.

Letelier S. (2001). Caleidoscopio de la creatividad. Santiago, Chile: Universitaria.

López, R. (2008). Creatividad con todas sus letras. Universitaria, Santiago, Chile. Recuperado de http:/issuu.com/anzuelo/docs/ creatividad_con_todas_sus_letras/1

López R. (1999). Prontuario de la creatividad. 2a ed. Santiago, Chile: Bravo y Allende Editores,. Recuperado de http://issuu.com/anzuelo/ docs/prontuario

Maslow, A. (1990). La personalidad creadora. 4a ed. Barcelona, España: Kairos.

Morin, E. (2002). El método II. La vida de la vida. 5a ed. Madrid, España: Cátedra-Teorema. 
Morin, E. (2001). El método V. La humanidad de la humanidad. 3a ed. Madrid, España: Cátedra-Teorema.

Morin, E. (2001a). El método IV. Las ideas de las ideas. 5a ed. Madrid, España: Cátedra-Teorema.

Morin, E. (2001b). La mente bien ordenada. Barcelona, España: Seix Barral- Los Tres Mundos,.

Morin, E. (2000). El método I. La naturaleza de la naturaleza. 4a ed. Madrid, España: Cátedra-Teorema.

Morin, E. (1999). El método III. El conocimiento del conocimiento. $3^{\mathrm{a}}$ ed. Madrid, España: Cátedra-Teorema.

Morin, E. (1999a). Los siete saberes necesarios para la educación del futuro. París, Francia: Unesco.

Soto, M. (1999). Edgar Morin. Complejidad y sujeto humano (tesis de Doctorado). Universidad de Valladolid, España. Recuperado de http:// www.biblioteca.org.ar/libros/133208.pdf

Torres, L. C. (2007). Complejidad. Aspectos básicos. Bogotá, Colombia: Unidad de Publicaciones, Facultad de Ingeniería, Universidad Nacional de Colombia.

Torres, L. C. (2011). Creatividad en el aula. Colección 150 años Facultad de Ingeniería, Universidad Nacional de Colombia. Bogotá, Colombia: UN Editorial.

Torres, L. C. (2012). Creatividad, estímulos para su desarrollo. Bogotá, Colombia: Ediciones de la U.

Veraldi, G. y Veraldi, B. (1998). Psicología de la creación. 2a ed. Bilbao, España: Mensajero. 